\title{
CONCENTRAÇÃO MICELAR CRÍTICA E CONCENTRAÇÃO CRÍTICA DE COALESCÊNCIA DE REAGENTES DE FLOTAÇÃO
}

\author{
A. S. BRAGA ${ }^{1 *}$, T. C. SOUZA PINTO ${ }^{2}$, P. H. L. S. MATAI ${ }^{1}$ e L. S. LEAL FILHO ${ }^{2}$ \\ ${ }^{1}$ Universidade de São Paulo (USP-SP). Departamento de Engenharia de Minas e de Petróleo \\ ${ }^{2}$ Instituto Tecnológico Vale - Mineração (ITV-MI) \\ andre.braga@usp.br*
}

Artigo submetido em dezembro/2015 e aceito em dezembro/2015

DOI: 10.15628/holos.2015.3824

\section{RESUMO}

A geração de bolhas de ar numa célula de flotação produz uma distribuição de tamanhos que pode ser representada pelo diâmetro médio de Sauter $\left(D_{3,2}\right)$, cuja magnitude diminui com a concentração de surfatante na solução até um valor crítico, denominado de concentração crítica de coalescência (CCC), sendo que este comportamento pode ser modelado através da equação de Laskowski. Este trabalho objetivou determinar a CCC, estimando as constantes de decaimento (B) e de redução do diâmetro de bolha (A) da equação de Laskowski. A concentração micelar crítica
(CMC) também foi determinada avaliando a tensão superficial em função da concentração de reagentes para dois espumantes e um coletor (com ação espumante). Os resultados permitiram comparar o potencial espumante dos reagentes estudados, indicando que o coletor estudado (Flotigam EDA), que também atua como espumante, apresentou CMC (80 ppm) maior que a CCC (8 ppm). O Mibcol apresentou a mesma ordem de CCC que o coletor ( $8 \mathrm{ppm}$ ) e a CCC do Flotanol D25 foi observada em concentração mais alta (15 ppm).

PALAVRAS-CHAVE: Flotação, espumante, concentração crítica de coalescência, concentração micelar crítica, diâmetro de Sauter.

\section{CRITICAL MICELLE CONCENTRATION AND CRITICISM CONCENTRATION OF FLOTATION REAGENT COALESCENCE}

\begin{abstract}
The generation of air bubbles in a flotation cell produces a size distribution which can be represented by Sauter mean diameter $\left(D_{3,2}\right)$, whose magnitude decreases as surfactant dosages increases in the bulk solution until a critical value, named as critical coalescence concentration (CCC), that obeys Laskowski's equation. This work aimed to determine the CCC, estimating through experimental work, the decay constant (B) and the bubble size reduction constant $(A)$ of Laskowski's
\end{abstract}

equation. The critical micelle concentration (CMC) was also determined evaluating the surface tension in function of reagent dosage for two frothers and 1 collector that also act as frother. The results allowed comparing the frother potential of the reagents studied, indicating that the collector presented $\mathrm{CMC}(80 \mathrm{ppm})$ greater than its CCC ( $8 \mathrm{ppm})$. The Mibcol has reached the same magnitude of the collector $(8 \mathrm{ppm})$ and Flotanol D25 presented a higher concentration (15 ppm) for CCC.

KEYWORDS: Froth flotation, frother, critical coalescence concentration, critical micelle concentration, Sauter mean diameter. 


\section{INTRODUÇÃO}

A água não é capaz de produzir espuma pelo fato de ser drenada instantaneamente no filme interbolhas, promovendo coalescência imediata das bolhas e consequente colapso. Espumantes são surfatantes formados por moléculas neutras contendo um ou mais grupos polares (hidroxila, éter, cetona) (Leja, 1982), desta forma o acréscimo destes reagentes fornece estabilidade às espumas da flotação formando uma camada de moléculas orientadas na interface líquido/gás das bolhas (parte apolar orientada à fase gasosa; parte polar orientada à fase líquida). Tal camada de moléculas adsorvidas nas bolhas estabiliza a espuma retardando a drenagem e aumentando seu volume, reduzindo a coalescência das bolhas (Melo e Laskowski, 2006).

A concentração crítica de coalescência (CCC) de um espumante corresponde à menor concentração que proporciona o menor diâmetro de bolha (Cho e Laskowski, 2002), podendo ser determinada graficamente como demonstrado na Figura 1 ou através da aproximação de dados experimentais (diâmetro médio de Sauter e concentração do espumante) à Equação 1. A CCC é considerada como um importante parâmetro para comparar o desempenho de diferentes espumantes de flotação (Laskowski, 2003; Nesset et al., 2007).

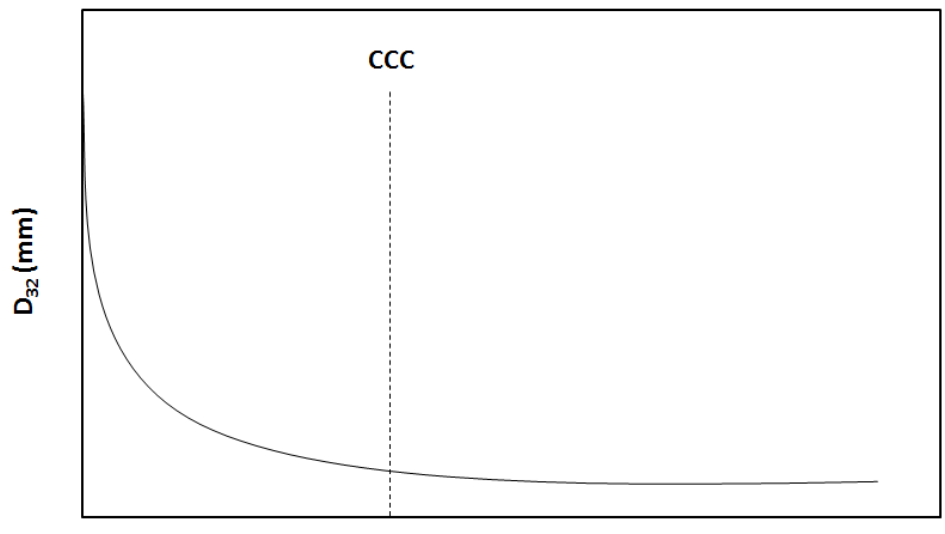

Frother Concentration (ppm)

Figura 1 - Determinação gráfica da CCC de um espumante (adaptado de Laskowski, 2003).

$D_{3,2}=d_{L}+A \cdot e^{(-B . C)}$

(Equação 1)

Sendo:

$\mathrm{D}_{3,2} \quad=$ Diâmetro de bolhas médio de Sauter $(\mathrm{mm})$;

$\mathrm{d}_{\mathrm{L}} \quad$ = Diâmetro de bolha mínimo $(\mathrm{mm})$;

A = Redução de diâmetro de bolha $(\mathrm{mm})$;

B = Constante de decaimento;

C = Concentração de espumante (ppm).

O diâmetro de bolha mais utilizado para representação de uma distribuição de diâmetros de bolhas é o diâmetro médio de Sauter e consiste na média da distribuição de bolhas no meio aquoso, conforme indica a Equação 2, sendo $\mathrm{n}_{\mathrm{i}}$ o número de bolhas em determinado diâmetro e $\mathrm{d}_{\mathrm{bi}}$ o diâmetro das bolhas, comumente gerando uma distribuição normal. 
$D_{3,2}=\frac{\sum n_{i} d_{b i}^{3}}{\sum n_{i} d_{b i}^{2}}$

(Equação 2)

A tensão superficial de um líquido $(\gamma)$ pode ser definida como a energia necessária para aumentar a superfície por unidade de área, sendo caracterizada como um trabalho isotérmico reversível. Por ser um fenômeno de superfície e depender de interações moleculares do bulk de uma solução, a tensão superficial é fortemente influenciada pela temperatura e pelo aumento da concentração de surfatantes. A maioria dos surfatantes provoca a redução da tensão superficial conforme as moléculas se arranjam na interface líquido/gás da superfície em formato de um filme difuso (Schambil e Schwuger, 1987). Esta redução da tensão superficial ocorre até um grau de compactação máximo do filme a partir do qual, ao invés de organizar-se na superfície, a espécie surfatante acrescentada à solução inicia a formação de agregados coloidais denominados micelas. A concentração mínima para que isto ocorra é chamada de concentração micelar crítica (CMC), sendo seu valor absoluto uma referência para quantificação da dosagem de reagentes na flotação por espuma. $\mathrm{O}$ valor de CMC pode sofrer influência de diversos aspectos como comprimento de cadeia, adição de eletrólitos ou ainda presença de impurezas (Rao, 2004; Rosen, 1989).

\section{MATERIAL E MÉTODOS}

\subsection{Materiais}

Os três reagentes foram selecionados para determinação de CMC e CCC, sendo apresentados na Tabela 1. É importante destacar a escolha de dois espumantes (Mibcol ${ }^{\oplus}$ e Flotanol D25 ${ }^{\circ}$ ) e um coletor com ação espumante (Flotigam EDA ${ }^{\circ}$ ) para os estudos comparativos deste trabalho.

Tabela 1. Reagentes utilizados para estudos de CMC e CCC.

\begin{tabular}{|c|c|c|c|c|}
\hline $\begin{array}{c}\text { Nome } \\
\text { Comercial }\end{array}$ & Funcionalidade Química & Aplicação na Flotação & Pureza & Fabricante \\
\hline Mibcol & metilisobutilcarbinol & espumante & $\begin{array}{c}\text { analítica } \\
(99 \%)\end{array}$ & Solvay/Rhodia \\
\hline Flotigam EDA & dodeciléter amina & $\begin{array}{l}\text { coletor catiônico } \\
\text { /espumante }\end{array}$ & comercial & Clariant \\
\hline Flotanol D25 & polipropileno glicol & espumante & $\begin{array}{c}\text { analítica } \\
(99 \%)\end{array}$ & Clariant \\
\hline
\end{tabular}

\subsection{Métodos}

\subsubsection{Medidas de tensão superficial}

Para os estudos de determinação da concentração micelar crítica e de concentração crítica de coalescência, os três reagentes foram preparados em solução aquosa, medindo-se a dosagem em ppm (relação massa/massa).

O equipamento utilizado para determinação da tensão superficial em função da concentração das soluções foi o Tensiômetro Krüss (K12), optando-se por medir a tensão 
superficial das soluções através da placa de platina (método de Wilhelmy) à temperatura constante de 25 으.

\subsubsection{Medidas de diâmetro de bolhas}

Para determinação dos diâmetros de bolhas em função da concentração das soluções, utilizou-se um aparato experimental que consistia em acoplar a sonda de um medidor de tamanho de bolhas APBS (desenvolvido pela Universidade da Cidade do Cabo - UCT) em uma célula de flotação de bancada Engendrar (CFB 1000N). Para todas as medições de diâmetro de bolhas manteve-se a célula de flotação em rotação constante de $1300 \mathrm{rpm}$ com vazão de ar em 4,2 L/min $\left(7.10^{-5} \mathrm{~m}^{3} / \mathrm{s}\right.$ no S.I.). $O$ medidor de bolhas APBS captura fotos de bolhas ascendentes à sonda (Figura 2a) e um software analisa as imagens calculando o diâmetro médio de Sauter $\mathrm{D}_{3,2}$ (Figura $2 b)$.

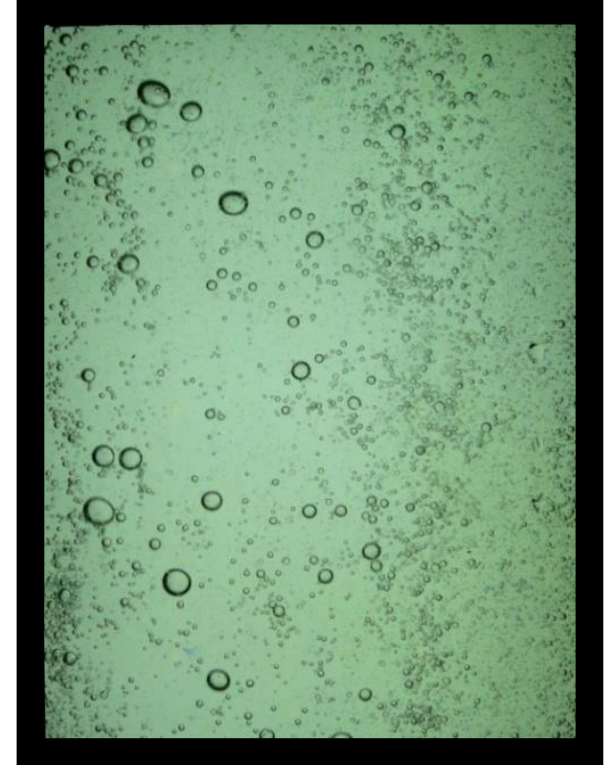

Figura 2a - Bolhas ascendentes (Mibcol, 9 ppm) no medidor APBS (UCT).

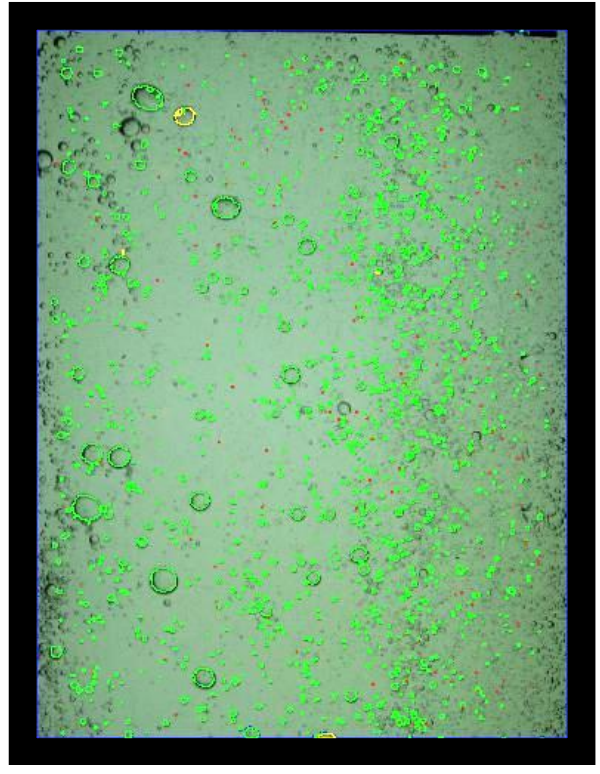

Figura $2 \mathrm{~b}$ - Tratamento de imagens pelo software APBS (UCT).

\section{RESULTADOS E DISCUSSÃO}

Os resultados de tensão superficial $(\mathrm{mN} / \mathrm{m})$ em função da concentração $(\mathrm{ppm})$ dos três reagentes são apresentados na Figura 3 e na Figura 4. De acordo com a Figura 3, observa-se que os espumantes Mibcol e Flotanol D25 não apresentaram formação de micelas na faixa de concentração avaliada. Também foi observado que os espumantes apresentaram influência na tensão superficial da solução à medida que um aumento da sua dosagem apresentou a redução da magnitude de $\gamma$, reportando também um aspecto de surfatante. 


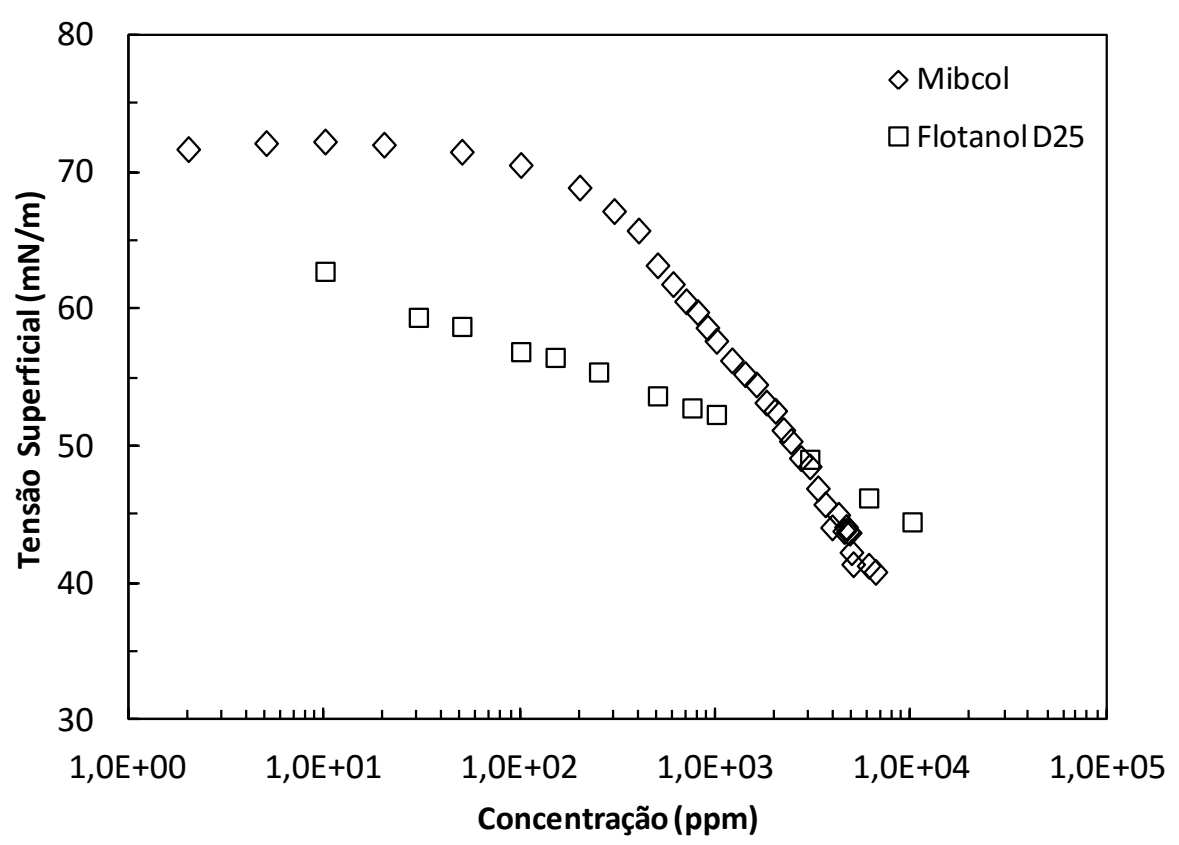

Figura 3 - Tensão superficial versus concentração dos espumantes Mibcol e Flotanol D25.

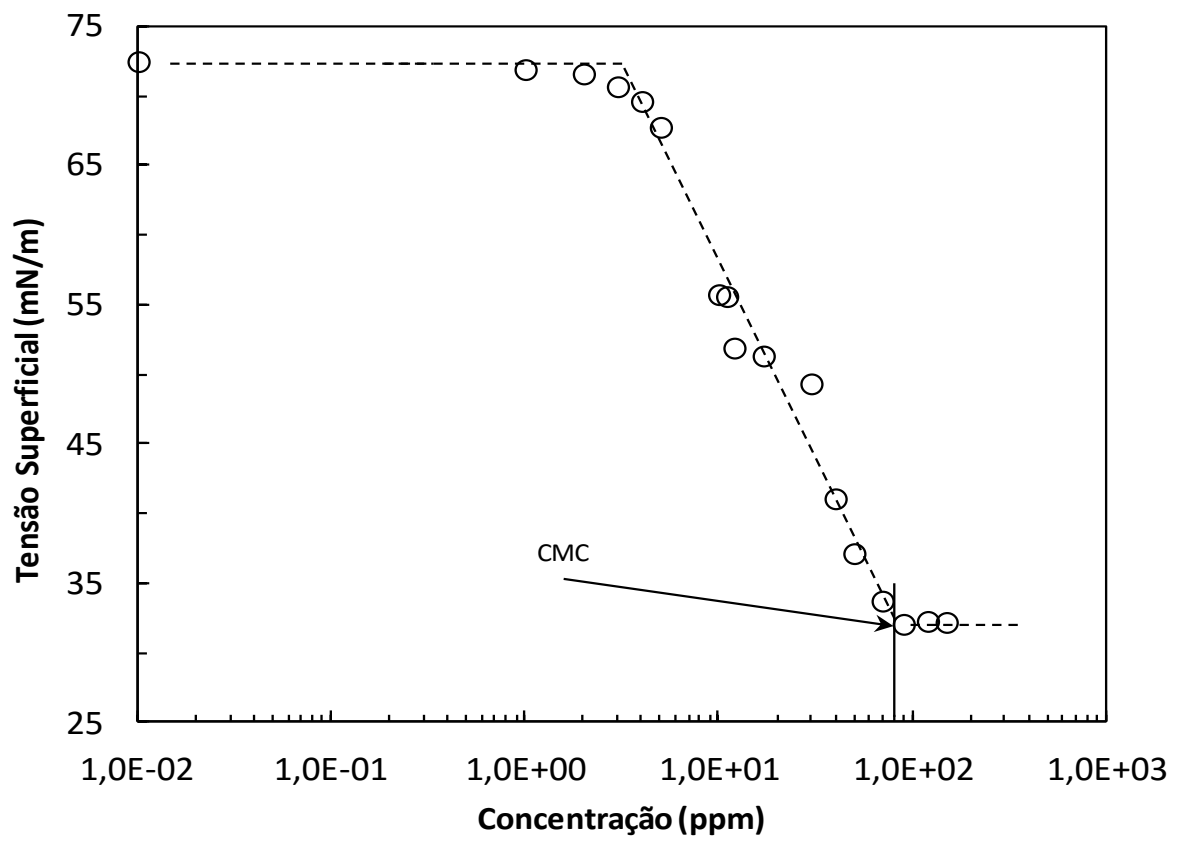

Figura 4 - Influência da concentração de Flotigam EDA na tensão superficial líquido/gás.

Observando a Figura 4, verifica-se que o surfatante Flotigam EDA apresentou formação de micelas a partir da concentração de 80 ppm. Devido ao fato de ser um reagente de pureza comercial, após a linearização dos dados em escala mono-log é possível compreender o desvio de alguns pontos do segmento inclinado como efeito de impurezas naturais de um reagente de caráter industrial. 
A Figura 5 compara a influência da concentração no diâmetro médio de Sauter $\left(D_{3,2}\right)$ das bolhas na célula de flotação de bancada experimental para os reagentes estudados. É possível observar que conforme a concentração aumenta o diâmetro das bolhas diminui como consequência direta da redução do efeito de coalescência.

De acordo com os dados apresentados na Figura 5, observa-se que os reagentes que apresentam menor CCC (Mibcol e Flotigam EDA) são aqueles que demonstraram desempenho espumante destacável, pois alcançam o diâmetro mínimo de bolhas com menores concentrações (8 ppm). O valor de 8 ppm obtido para o Mibcol coincide com o reportado por Cho e Laskowski (2002).

O Flotanol D25 também apresentou CCC adequada (15 ppm), embora sua concentração crítica de coalescência seja quase duas vezes maior que do Mibcol e do Flotigam EDA.

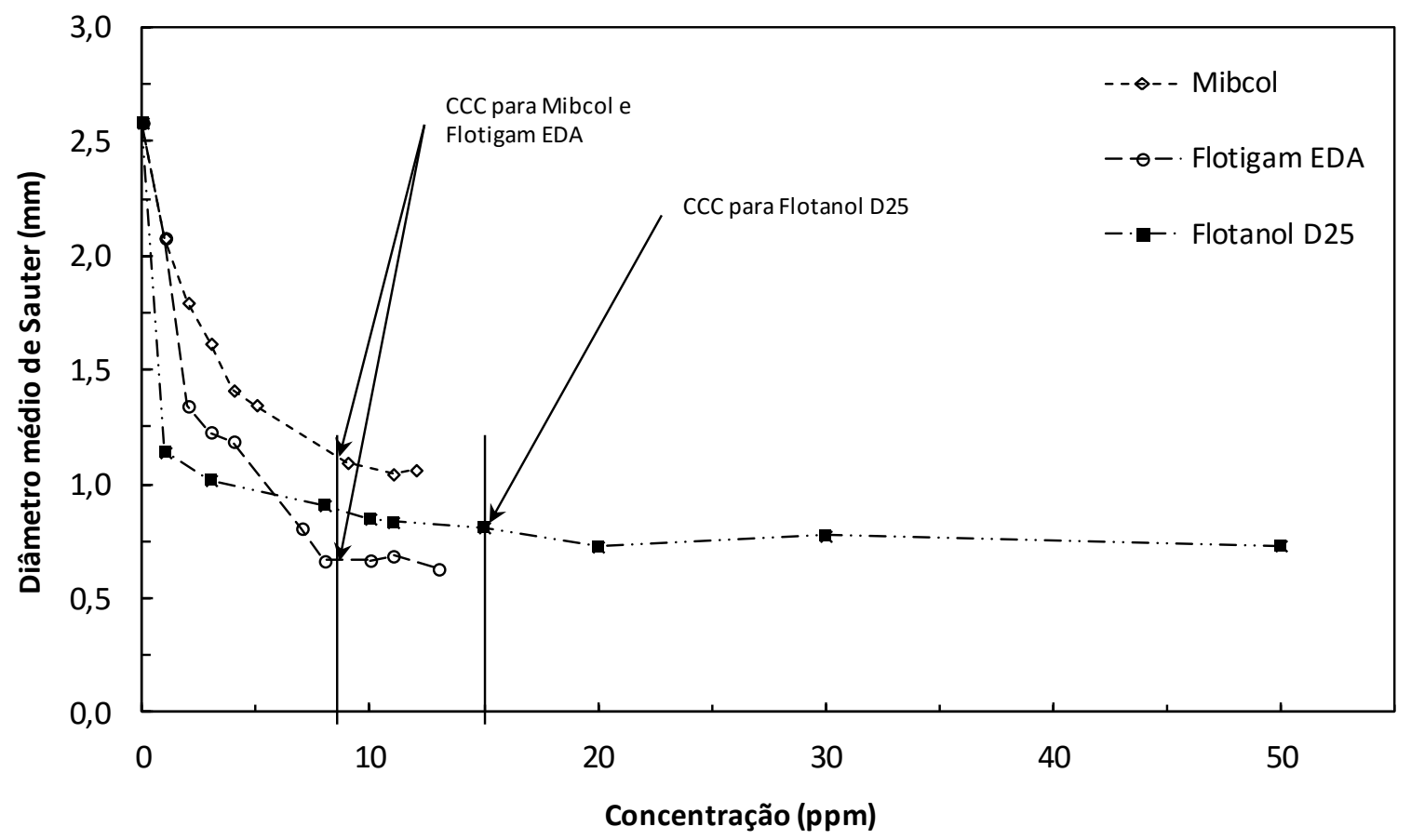

Figura 5 - Concentração crítica de coalescência para os reagentes estudados.

Através das medidas obtidas de CCC, é possível aproximar os dados experimentais ao modelo de Laskowski proposto na Equação 1 e obter os parâmetros de diâmetro de bolha limite $\left(d_{L}\right)$, redução de diâmetro $(A)$ e constante de decaimento $(B)$ para cada reagente estudado. Os resultados experimentais e os parâmetros da equação de Laskowski obtidos são apresentados na Tabela 2.

De acordo com os dados explicitados na Tabela 2, é possível observar que o Mibcol apresenta comportamento característico de um espumante: CCC pequena e alta redução de diâmetro (A). O outro espumante estudado neste trabalho (Flotanol D25) apresentou maior CCC (15 ppm), embora observa-se que a constante de decaimento (B) tenha sido a mais pronunciada, dando indicativos de que em menores concentrações ( $\leq 5 \mathrm{ppm}$ ) este reagente consiga ser mais eficiente na prevenção da coalescência das bolhas. Esta afirmativa pode ser comprovada 
experimentalmente conforme indicado na Figura 5, na qual em concentrações inferiores a 5 ppm os diâmetros de bolha foram menores para o Flotanol D25.

Tabela 2 - CMC, CCC e parâmetros de Laskowski $d_{L}$, A e B (*) para os reagentes estudados.

\begin{tabular}{cccccc}
\hline $\begin{array}{c}\text { Nome } \\
\text { Comercial }\end{array}$ & $\begin{array}{c}\text { CMC } \\
(\mathrm{ppm})\end{array}$ & $\begin{array}{c}\mathrm{CCC} \\
(\mathrm{ppm})\end{array}$ & $\begin{array}{c}\text { Diâmetro de bolha } \\
\text { limite } \mathrm{d}_{\mathrm{L}}(\mathrm{mm})\end{array}$ & $\begin{array}{c}\text { Redução de } \\
\text { diâmetro A }(\mathrm{mm})\end{array}$ & $\begin{array}{c}\text { Constante de } \\
\text { decaimento B }\end{array}$ \\
\hline Mibcol & - & 8 & 0,987 & 1,576 & 0,327 \\
Flotigam EDA & 80 & 8 & 0,639 & 1,959 & 0,391 \\
Flotanol D25 & - & 15 & 0,828 & 1,754 & 1,668 \\
\hline
\end{tabular}

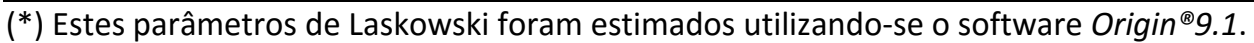

O Flotigam EDA é um coletor que apresentou menor CCC e maior redução de diâmetro A (menor $\mathrm{d}_{\mathrm{L}}$ ). Como de forma geral, a prática industrial para coletores de flotação é utilizar dosagens menores aos valores de CMC. Desta forma é possível observar que o reagente opera com o diâmetro bolha limite de $0,639 \mathrm{~mm}$ e o valor da concentração do reagente para atingir a CCC (8 ppm) é dez vezes menor do que para a formação de micelas (80 ppm).

\section{CONCLUSÕES}

Parâmetros de CMC e CCC dos três reagentes estudados foram determinados, assim como as constantes $-d_{L}$, A e B - da equação de Laskowski. A partir dos resultados experimentais foi possível comparar o potencial espumante dos reagentes testados, verificando que o Mibcol e o Flotigam EDA apresentaram menor CCC que o Flotanol D25. Em contrapartida, o Flotanol D25 mostrou-se mais eficiente por apresentar maior constante de decaimento (B).

\section{AGRADECIMENTOS}

Os autores gostariam de agradecer ao ITV-MI e a equipe do LFQI-USP.

\section{REFERÊNCIAS}

1. Cho, Y.S.; Laskowski, J.S. Effect of flotation frothers on bubble size and foam Stability. Int. J. of Mineral Processing, 2002; 64; 69-80.

2. Laskowski, J.S. Fundamental properties of flotation frothers. Proceedings 22nd Int. Mineral Processing Congress. The South African Institute of Mining and Metallurgy, 2003, Cape Town, South Africa. p. 788-797.

3. Leja, J. Surface chemistry of froth flotation. Plenum Press, New York; 1982.

4. Melo, F.; Laskowski, J.S. Fundamental properties of flotation frothers and their effect on flotation. Minerals Engineering, 2006; 19; 766-773.

5. Nesset, J.E.; Finch, J.A.; Gomez, C.O. Operating variables affecting the bubble size in forced-air mechanical flotation machines. Proceedings AusIMM 9th Mill Operators' Conference, 2007, Fremantle, Australia. p. 66-75.

6. Rao, S. R. Surface chemistry of froth flotation - Rev. ed. of: Surface chemistry of froth flotation/ Leja, J. c1982 - $2^{\text {nd }}$ ed., V.1; New York, 2004. Chapter 4, Physical chemistry of interfaces, $p$. 143-208. 
7. Rosen, M. J. Surfactants and interfacial phenomena. $2^{\text {nd }}$ ed.; New York, 1989. Chapter 2-5, p. 33-229.

8. Schambil, F.; Schwuger, M.J. Surfactants in consumer products. Springer-Verlag, Berlin, 1987; Chapter 4, Interfacial and colloidal pr 